\title{
Belohnung für Fachärzte, die freie Termine melden
}

Fachärzte in Niedersachsen erhalten Honorarvorteile, wenn sie der KV freie Termine zur Vermittlung in den Terminservicestellen melden. Das Modell könnte eine Blaupause auch für andere KV-Bezirke werden.

Ein kleiner Anreiz soll helfen: In Niedersachsen können je Facharzt bis zu 24 Termine pro Quartal von den Honorarbegrenzungen ausgenommen werden, sofern diese Termine an die Terminservicestelle der KV Niedersachsen gemeldet wurden. Das hat die Vertreterversammlung beschlossen. Dazu müssen die entsprechenden Termine bei der Abrechnung gekennzeichnet werden. Die KV rechnet mit einem jährlichen Volumen von 1,5 bis 2 Millionen $€$. „So wollen wir Anreize schaffen, dass genug Termine gemeldet werden", kommentiert KV-Sprecher Detlef Haffke die Regelung. Vor allem bei Augenärzten, Kinder- und Jugendpsychiatern, Neurologen, Orthopäden, Pneumologen, Radiologen mit MRT und Rheumatologen könnte es eng werden, sodass freie Termine dort besonders begehrt sein werden. Die KV geht von rund 18 Millionen Überweisungen im Jahr und einer Vermittlungsquote von 2 bis $3 \%$ pro Jahr aus. Das wären rund 500.000 Patienten jährlich, die über die Terminvermittlungsstelle Niedersachsens zu einem Facharzt kommen. Wie sehr die Terminvermittlung tatsächlich in Anspruch genommen werden wird, muss man natürlich abwarten. Das System greift erst seit dem 25. Januar.

Und so funktioniert die Vermittlung: Die Ärzte melden ihre freien Termine online. Patienten geben ihre Termine ebenfalls online an oder rufen das Callcenter der KV Niedersachsen an. Nur bei vorliegender Überweisung können Patienten den Service in Anspruch nehmen. Dazu werden die zu vermittelnden Überweisungen mit einem zwölfstelligen Code gekennzeichnet - zunächst per Aufkleber, welche die KV an ihre Mitglieder schickt, später dann über die Praxissoftware.

„Diese Überweisungen werden vor allem die Hausärzte ausstellen, wenn sie beim Patienten eine Notwendigkeit für einen Facharztbesuch sehen", sagt Haffke. Nur mit diesem Code können sich Patienten dann telefonisch über das KV-Callcenter oder online einen Termin vermitteln lassen. „Allerdings können wir das Überweisungsverhalten der Hausärzte überhaupt nicht einschätzen oder vorhersagen“, betont Haffke.

Christian Beneker

Bei einer Urethralänge von über $8 \mathrm{~cm}$ und/oder nicht überwiegend interner Lage der Urethra ist die GOP 26310 berechnungsfähig. Bei überwiegend interner Lage der Urethra und einer Länge von bis zu $8 \mathrm{~cm}$ ist hingegen die GOP 26311 oder 08311 anzusetzen.

\section{- Erste Telemedizinziffer im EBM \\ Rund zwei Jahre haben die Ärz-} te auf eine Telemedizinziffer im EBM gewartet. Den Aufschlag macht nun die Überwachung von Patienten mit einem Defibrillator oder CRT-System. Die Leistung wird ab April abrechenbar sein. Herzschrittmacherpatienten bleiben allerdings zunächst außen vor.

Die neue Leistung für Kardiologen ist mit der GOP 13554 belegt und mit 279 Punkten bewertet. Für Kinderkardiologen gibt es die GOP 04417 (511 Punk- te). Voraussetzung für die Abrechnung ist ein persönlicher Arzt-Patienten-Kontakt pro Jahr zur Überwachung des Implantats. Ein eventuell notwendiger telefonischer Arzt-Patienten-Kontakt kann mit der neuen GOP 01438 (88 Punkte) abgerechnet werden. Die Leistungen werden innerhalb der morbiditätsorientierten Gesamtvergütung honoriert.

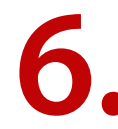

\section{Laborleistungen}

Für das erste Halbjahr 2016 beträgt die bundeseinheitliche Laborabstaffelungsquote „Q“ $91,58 \%$. Bei der Frage, welche Ärzte Laboruntersuchungen des EBM-Abschnitts 32.3 (Speziallabor) erbringen und abrechnen dürfen, gibt es allerdings erneut eine Schonfrist. Eigentlich sollte es hier bereits zum Januar eine neue Regelung geben, welche die Leistungserbringung vom Kern des Fachgebiets abhängig macht. Die Part- ner des Bundesmantelvertrages hätten diese Anpassung bislang jedoch nicht abschließend prüfen können, berichtet die KBV. Die Frist zur Anpassung wurde daher auf den 1. Juli 2016 und damit zum dritten Mal verschoben.

\section{7.} Neue Onkologieziffern

Die Tumordiagnostik mittels Positronen-Emissions-Tomografie (PET sowie PET/CT) kann nun über den EBM abgerechnet werden (GOP 34700 bis 347003). Die Leistung wird laut KBV außerhalb der morbiditätsorientierten Gesamtvergütung und damit extrabudgetär zu festen Preisen honoriert. Für Sachkosten im Zusammenhang mit den neuen Leistungen können Ärzte die ebenfalls neue Kostenpauschale 40584 (255 €) bei Verwendung des Radionuklids 18F-Fluordesoxyglukose berechnen.

\section{8.} Fristverlängerung für Hörgeräteversorgung

Der Bewertungsausschuss hat noch 2015 die Gültigkeit seiner Beschlüsse zur Hörgeräteversorgung verlängert. Damit können Ärzte die erst 2012 neu eingeführten EBM-Ziffern für die entsprechenden Leistungen nun bis Ende 2017 abrechnen.

Die Ziffern in den Kapiteln 9 (HNO) und 20 (Phoniatrie und Pädaudiologie) des EBM betreffen vor allem die Neuanpassung, Kontrolle und Nachsorge von Hörgeräten bei Kindern und Erwachsenen (GOP 09372 bis 09375,20338 bis 20340, 20372 bis 20375, 20377 und 20378). Aber auch einige Zusatzpauschalen, zum Beispiel für die Behandlung von Patienten mit schwer stillbarem Nasenbluten oder die Diagnostik des Tinnitus, fallen unter die Fristverlängerung, so die KBV.

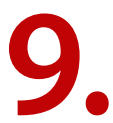

\section{Impfausweise}

Seit Januar müssen die Krankenkassen die Kosten für die Erstellung und den Versand von Impfausweisen übernehmen. So sieht es das Präventionsgesetz, das am 25. Juli 2015 in Kraft getreten ist, vor. Hier sollten Ärzte bei ihrer KV nachfragen. Zwar sollen die Krankenkassen das Vorgehen regeln, dies könnte aber ebenso Bestandteil der regionalen Impfvereinbarungen sein.

Rebekka Höhl 